\title{
The booking problem of a diagnostic resource with multiple patient classes and emergency interruptions
}

\begin{abstract}
CT department is an appointment based service system. All patients except emergency patients should be scheduled in advance. Regular patients arrive on time. Emergency patients arrive randomly and get non-preemptive priority. So the direct waiting time of regular patients should be explicitly described. The unit direct waiting cost gradually increases with the previous waiting time. On the one hand, too many regular patients will increase the direct waiting cost for them and overtime cost for medical resources. On the other hand, too few regular patients will cause idling of medical resources and penalty for delaying regular patients' requests. So a reasonable reservation level should be determined in advance. The booking problem is modeled as a finite horizon Markov decision process. Some booking limit structures are given to improve the total expected reward. The numerical analysis shows that our policies are much better than the policy currently employed.
\end{abstract}

Key Words: healthcare operations management; revenue management; emergency interruptions; marginal analysis.

Mathematics Subject Classification: $90 \mathrm{C} 40 \cdot 60 \mathrm{~K} 30$

\section{Introduction}

This work is originally motivated by a problem faced by Sichuan Provincial People's Hospital $(\mathrm{SCPH})$, which is a large-size hospital in China. After interviewing the medical staff and administrator, we recognize that CT scans are a bottleneck for providing patients timely service. CT scans include plain scan, enhancement scan and other types of scan. We focus on the plain scan. Reasons are listed below. Firstly, enhancement scan and other types CT scans are assigned to a specific machine because of the specific preparation work and operation procedure. Secondly, emergency patients only need plain scan. Thirdly, plain scan makes up $80-85 \%$ of the CT scans.

CT department is an appointment based service system. All patients except emergency patients should be scheduled in advance. No walk-in regular patients are allowed. Regular patients can be grouped into three categories: outpatient (OUT), inpatient (IN) and healthy patient for a checkup (HP, Health Management Center). Each workday is divided into two independent half-day sessions (8:00-12:00 and 14:00-18:00, similar half-day session has been considered in Sickinger and Kolisch, 2009). The basic time unit is a half-day service session. There are several identical service sessions opening for booking simultaneously. Each regular patient asks for a preferred service session when he makes an appointment. The scheduler will give the first available time on or after his preferred service session. Patients may be delayed

(C) 2017. This manuscript version is made available under the Elsevier user license http://www.elsevier.com/open-access/userlicense/1.0/ 
from the time at which they request. Regular patients whose booking requests are delayed will not leave but accept a later time which is given by scheduler (Gupta and Wang 2008; Dobson et al. 2011). This is in line with the practice in CT department. If the patients leave and choose to have their scan done elsewhere, they firstly enroll with other physicians and then make an appointment with CT department elsewhere. That may be more time-consuming and not cost-effective.

There are 3 parallel plain scanners in SCPH. The CT department currently allocates one scanner for the exclusive use for emergency patients. The utilization rate of the dedicated scanner is low, about $70 \%$ of its capacity. Other CT scanners for regular patients are in short supply. To better utilize the scarce resources, the dedicated scanner should be shared by regular patients and emergency patients. And emergency patients should have non-preemptive priority to service (Green et al. 2006; Luo et al. 2012). The cut in line of emergency patients will cause direct waiting time of regular patients. So a reasonable reservation level for emergency patients should be determined in advance. On the one hand, too many regular patients will increase the direct waiting time for them and the overtime working for medical resources. On the other hand, too few regular patients will cause idling of medical resources and delaying regular patients' booking requests frequently. The direct waiting time is the difference between a patient's appointment time and the time when he is actually served by the service provider (Gupta and Denton 2008).

The problem faced in CT department turns into the booking problem that how many requests arising in the booking horizon should be accepted to improve the total expected reward for a particular service session. The booking horizon is the time between its opening time and its closure time where the latter coincides with the beginning of the service session.

The major objective of this paper is to give a reasonable booking limit number for each regular patient taking into account the direct waiting cost for them. Complexity comes from the direct waiting cost of regular patients in the service session caused by random emergency arrivals. Specially, this paper makes the following contributions: we model the directing waiting time of the regular patients in the service session, as well as the unit direct waiting cost gradually increases with the previous waiting time. We establish the structural properties which lead to the booking limit structure in this paper. The booking limit number for each patient beyond which it is not optimal to book additional appointments is obtained by marginal analysis. The complex Markov decision process (MDP) model can be reduced to simple formulas regarding revenue and costs.

The primary application area of this work lies in healthcare. Moreover, this work could apply to several other areas if the service system has the following features. First, the service system is an appointment based service system. Second, the service system faces two types of independent customers, regular customers who are served on a FCFS basis and emergency customers who have higher access to service.

The rest of this paper is organized as follows. In section 2, we review the related literature. Section 3: we formulate the booking problem. Section 4 establishes structural properties. Section 5 reports the numerical results of different policies. A discussion for future research is given in section 6 .

\section{Literature Review}

Our analysis of this booking problem shares some traits with previous researches. Gupta and Wang (2008) determine the optimal booking policy for a primary-care clinic, where patients may have a preference for physician and date of service. Their objective is to maximize the 
revenue of a primary-care clinic, via marginal analysis on the expected benefit and expected loss of having one more slot available for same-day patients. Dobson et al. (2011) formulate a stochastic model of appointment scheduling in a primary health care practice. Direct waiting time is not considered in these two works since same-day patients (walk-in patients) accept any available slot on the workday. However, it does not hold in our work. We begin with a simple example below to highlight this point. More-realistic examples are presented later in this paper.

Suppose that there are 5 slots in one service session and each patient occupies one slot. Two regular patients arrive at the 1st and the 2nd slot according to the appointment time, respectively. Two emergency patients randomly arrive during the service session. There is no direct waiting time for regular patients if these emergency patients arrive after the 2nd slot. However, there exists direct waiting time if one of the emergency patients arrives before the 2nd slot. Mathematical modelling the direct waiting time and giving some structure properties which lead to booking limit structure for regular patients is one of the major features in this paper. Furthermore, our model could deal with arbitrary patient classes while there are two patient classes (Gupta and Wang 2008; Dobson et al. 2011).

Direct waiting time has been considered in these works (Green et al. 2006; Kolisch and Sickinger 2008; Sickinger and Kolisch 2009). These works are classified into allocating scheduling which schedules patients not in the booking horizon but in the service session (Magerlein and Martin 1978; Patrick et al. 2008). Both of these works focuses on determining which patient to serve next at each slot, given an arbitrary outpatient appointment schedule. Decisions are made at each slot in the service session. However, our work is classified into advance scheduling which decisions are made not in the service session but in the booking horizon. The booking limit number for each regular patient which is closely related to the direct waiting time in the service session should be given in the booking horizon.

Moreover, the direct waiting cost per unit time in different environments has been shown to gradually increasing with the previous waiting time (Osuna 1985; Carmon et al. 1995). We give two example scenarios to highlight this point. Scenario 1: a regular patient had been delayed 10 minutes. He was told to wait for an additional 5 minutes. Scenario 2: the same patient had been delayed 1 hour. He was told to wait for an additional 5 minutes. The same additional waiting time, the direct waiting cost is obviously not the same. The conventional constant delay cost in these works (Green et al. 2006; Kolisch and Sickinger 2008; Sickinger and Kolisch 2009) could not reflect this relationship. The exponential function is an effective form to measure this nonlinear relationship under different setting (Ittig 1994; Carmon et al. 1995). So an exponential function is employed to capture the unit direct waiting cost increasing with the previous waiting time in this work.

\section{$3 \quad$ Model description}

We focus on a particular service session (similar to Gupta and Wang 2008) and model the booking problem as a MDP. Firstly, we make the following operating assumptions in this work:

- A1. Regular patients arrive on time.

- A2. There are $c$ identical slots in each service session for each scanner. Each patient occupies one slot.

- A3. Regular patients are divided into $M$ classes.

- A4. There is no spillover of unfinished regular patients from one service session to the next.

Assumption A1 means that no-shows and unpunctual patients are not considered. Modelling the direct waiting time of regular patients becomes intractable due to the curse of dimensionality 
if we take into account these factors under the random demand of emergency patients. Patients in $\mathrm{CT}$ department of SCPH usually show up according to the appointment time.

The identical-length service slots reflects common practice in the previous literatures in MDP (Green et al. 2006; Gupta and Wang 2008; Patrick et al. 2008; Kolisch and Sickinger 2008; Sickinger and Kolisch 2009; Dobson et al. 2011).

The scheduler equally assigns regular patients to 3 parallel plain scanners in the booking horizon. Each regular patient will be given a specific time to come and assigned to one scanner. The triage nurse equally assigns emergency patients to 3 parallel plain scanners during the service session. So it is sufficient to consider one scanner's booking problem since 3 plain scanners are identical due to Assumption A2 and the fact that emergency patients only need plain scan.

Regular patients are divided into $M$ classes since the revenue depends on the the number of scan positions and the preference of the hospital. We use $r_{m}(1 \leqslant m \leqslant M)$ to represent the total revenue of receiving a $m$ th class patient. All emergency patients should be accommodated. The ability to serve emergency patient will increase the reputation of the hospital. The revenues for emergency patients is not included in the model (Green et al. 2006; Sicking and Kolisch 2008). $\pi_{m}(1 \leqslant m \leqslant M)$ represents an average estimation of the penalty cost for delaying the $m$ th patient class's request.

Assumption A4 is a typical practice in hospital. CT department has to work until all the delayed regular patients being accomplished after service session. Due to the cost of running the scanner (facilities, equipment and staff salaries), overtime cost occurs. Let $\gamma_{0}$ be the overtime cost per slot for exceeding the service session's capacity. There is no emergency interruption after regular hours since emergency demand will be served by acute care unit after regular hours. Furthermore, we use $\pi_{0}$ to denote the penalty for an unutilized slot (Gupta and Wang 2008).

Drawing upon ideas from revenue management, the booking horizon is divided into $\tau$ discrete decision periods, indexed by $t=\tau, \tau-1, . ., 1$, such that the scheduler deals with at most one booking request in each period. Obviously, the length of each decision period is not the same since the interval arrival time of booking requests is not the same. The scheduler can observe the reservation state and the patient class of booking request at the beginning of each decision period. The probability that a $m$ th class patient requests an appointment in period $t$ is $\lambda_{t}^{m}$. $\lambda_{t}$ $\left(\lambda_{t}=\sum_{m=1}^{M} \lambda_{t}^{m} \leqslant 1\right)$ is the call rate in period $t$. Notation $(\cdot)^{+}$stands for $\max \{\cdot, 0\}$ and $a \bigwedge b$ stands for $\min \{a, b\}$.

Let $v_{t}(j)$ be the maximum total expected reward obtained from period $t$ to 1 , given the reservation state at time $t$ is $j, j \in(0, c)$. The service system achieves $v_{t}(j)$ by choosing the best accept/delaying decision for each $t$ and each $j$. Its maximum total expected reward is $v_{\tau}(0)$, where 0 represents all the slots are unreserved when booking opens. Then the appointment problem can be reduced to recursively solving the following optimality equations for $t=\tau, \tau-$ $1, \ldots, 1$ :

$$
\begin{aligned}
v_{t}(j) & =\sum_{m=1}^{M} \lambda_{t}^{m} \max \left\{v_{t-1}(j+1)+r_{m}, v_{t-1}(j)-\pi_{m}\right\}+\left(1-\sum_{m=1}^{M} \lambda_{t}^{m}\right) v_{t-1}(j) \\
& =\sum_{m=1}^{M} \lambda_{t}^{m} \max \left\{\Delta v_{t-1}^{m}(j), 0\right\}+v_{t-1}(j)-\sum_{m=1}^{M} \lambda_{t}^{m} \pi_{m} .
\end{aligned}
$$

In (1), the term $\Delta v_{t-1}^{m}(j)=v_{t-1}(j+1)-v_{t-1}(j)+r_{m}+\pi_{m}$ denotes the incremental benefit of reserving the $(j+1)$ th slot to a $m$ th class patient at time $t$ when the reservation state is $j$ $(\max \{A, B\}=\max \{A-B, 0\}+B)$. A $m$ th class patient should be scheduled the $(j+1)$ th slot at time $t$ if and only if $\Delta v_{t-1}^{m}(j)>0$. 
The service session starts at $t=0$. Emergency demand occur randomly during the service session. No more than one emergency patient could be assigned to each slot since the service time is equal to the slot length. Let $X_{i} \in\{1,0\} \quad(1 \leqslant i \leqslant c)$ denote the random number of emergency patient in the $i$ th slot,

$$
\left\{\begin{array}{l}
P\left(X_{i}=1\right)=p_{i} \\
P\left(X_{i}=0\right)=1-p_{i} .
\end{array}\right.
$$

Namely, the random arrival of emergency patient is described by a sequence of independent and non-identical Bernoulli variables. $Y_{i}=\sum_{k=1}^{i} X_{k}$. $Y_{i}$ denotes the random number of emergency patients until the beginning of the $i$ th slot.

For the calculation of the direct waiting cost, we need to introduce indicator functions $M_{i}(j)$ $(i=1,2, \ldots, c)$

$$
M_{i}(j)=\left[1 \wedge\left(Y_{i}+j-i\right)^{+}\right]= \begin{cases}1, & \text { if } Y_{i}+j>i \\ 0, & \text { if } Y_{i}+j \leqslant i,\end{cases}
$$

where $M_{i}(j)=1$ if the number of emergency patients until the $i$ th slot and the total number of regular patients combined is larger than current slot $i$, and $M_{i}(j)=0$ otherwise. There are $Y_{i-1}$ emergency patients cutting in line before the $i$ th slot. The number of regular patients who complete service is $i-1-Y_{i-1}$ if $j \geqslant i-1-Y_{i-1}$. An emergency patient occupying the $i$ th slot will result in each of the rest $\left(j-i+1+Y_{i-1}\right)^{+}$regular patients delaying one more slot according to Assumption A1. Note that each of the rest $\left(j-i+1+Y_{i-1}\right)^{+}$regular patients has been delayed $Y_{i-1}$ slots. The unit direct waiting cost per patient who has been delayed $Y_{i-1}$ slots is given by an exponential function (Ittig 1994; Carmon et al. 1995) of the form $e^{a Y_{i-1}+b}(a \geqslant 0)$. Obviously, $Y_{0}=0$.

Let $W_{i}\left(Y_{i}, X_{i}, j\right)$ denote the direct waiting cost due to the $i$ th slot occupied by an emergency patient. Then

$$
W_{i}\left(Y_{i}, X_{i}, j\right)=M_{i}(j) \cdot X_{i} \cdot\left(j-i+1+Y_{i}-X_{i}\right) \cdot e^{a Y_{i-1}+b}
$$

The total direct waiting cost in one service session is $W(j)=\sum_{i=1}^{c} W_{i}\left(Y_{i}, X_{i}, j\right)$.

With the above notation, the expected cost from service session is

$$
v_{0}(j)=-\pi_{0} E\left[\left(c-j-Y_{c}\right)^{+}\right]-\gamma_{0} E\left[\left(Y_{c}+j-c\right)^{+}\right]-E(W(j)),
$$

where $E(W(j))=\sum_{i=1}^{c} E_{Y_{i}, X_{i}}\left(W_{i}\left(Y_{i}, X_{i}, j\right)\right)$ is the expected direct waiting cost caused by random demand of emergency patients in the service session.

To see the idea of calculating process of the direct waiting cost, we present two simple examples in this section.

Example 1. Suppose there are 12 slots in one session and 4 regular patients. In the service session, the random demand of emergency patients is $X_{1}=1, X_{2}=0, X_{3}=1, X_{4}=1, X_{5}=0$, $X_{6}=0, X_{7}=0, X_{8}=1, X_{9}=1, X_{10}=0, X_{11}=0, X_{12}=1$. Then $Y_{1}=1, Y_{2}=1$, $Y_{3}=2, Y_{4}=3, Y_{5}=3, Y_{6}=3, Y_{7}=3, Y_{8}=4, Y_{9}=5, Y_{10}=5, Y_{11}=5, Y_{12}=6$. According to (3)-(4), the direct waiting cost of each slot is $W_{1}(1,1,4)=4 e^{b}, W_{2}(1,0,4)=0$, $W_{3}(2,1,4)=3 e^{a+b}, W_{4}(3,1,4)=3 e^{2 a+b}, W_{5}(3,0,4)=0, W_{6}(3,0,4)=0, W_{7}(3,0,4)=0$, $W_{8}(4,1,4)=0, W_{9}(5,1,4)=0, W_{10}(5,0,4)=0, W_{11}(5,0,4)=0, W_{12}(6,1,4)=0$. The total direct waiting cost $W(4)$ is $4 e^{b}+3 e^{a+b}+3 e^{2 a+b}$. 
Example 2. If the scheduler accepts one more patient (based on Example 1) at the decision period $t=1$ (5 regular patients ), then $W_{1}(1,1,5)=5 e^{b}, W_{2}(1,0,5)=0, W_{3}(2,1,5)=4 e^{a+b}$, $W_{4}(3,1,5)=4 e^{2 a+b}, W_{5}(3,0,5)=0, W_{6}(3,0,5)=0, W_{7}(3,0,5)=0, W_{8}(4,1,5)=e^{3 a+b}$, $W_{9}(5,1,5)=e^{4 a+b}, W_{10}(5,0,5)=0, W_{11}(5,0,5)=0, W_{12}(6,1,5)=0$. The total direct waiting cost $W(5)$ is $5 e^{b}+4 e^{a+b}+4 e^{2 a+b}+e^{3 a+b}+e^{4 a+b}$. The increased direct waiting cost is: $e^{b}+e^{a+b}+e^{2 a+b}+e^{3 a+b}+e^{4 a+b}$.

\section{Structural Properties}

We first prove the concavity of $v_{t}(j)$ in $j$. Then the booking limit numbers for different patient classes are obtained by solving a newsvendor-like problem. These threshold numbers ensure that the incremental benefit of each regular patient is positive at each decision period in the booking horizon. At last, we give improved booking actions on the total expected reward.

\subsection{Preliminary lemmas}

Firstly, let $H\left(Y_{c}, j\right)=-\pi_{0}\left(c-j-Y_{c}\right)^{+}-\gamma_{0}\left(Y_{c}+j-c\right)^{+}$.

Lemma 1. The function $H\left(Y_{c}, j\right)$ is concave in $j$.

The proofs of all results in this section are contained in the Appendix.

Lemma 2. The function $v_{0}(j)$ is concave in $j$.

$v_{0}(j+1)-v_{0}(j)$ is non-increasing in $j$ from Lemma 2. $v_{0}(j+1)-v_{0}(j)$ is related to the reservation state $j$, independent of the patient class. The incremental benefit $\Delta v_{0}^{m}(j)$ is related to both $j$ and $m$. For a given $m$ th class patient, $\Delta v_{0}^{m}(j+1) \leqslant \Delta v_{0}^{m}(j)$ at the decision period $t=1$.

Lemma 3. Suppose $v(j)$ is concave in $j \geqslant 0$, then $f(j)=\max \left\{v(j+1)+r_{m}, v(j)-\pi_{m}\right\}$ is concave in $j \geqslant 0$ as well.

We give the following theorem based on the above three lemmas.

Theorem 1. $v_{t}(j)$ is concave in $j$ for arbitrary decision period $t \in(\tau, \tau-1, \ldots, 1)$.

Theorem 1 shows that the total expected reward function is concave at arbitrary decision period for arbitrary reservation state. The booking limit structure is established on the basis of this important theorem.

\subsection{Booking limit structure}

Proposition 1. The incremental benefit $\Delta v_{t}^{m}(j)(\forall j, t)$ satisfies:

(1) $\Delta v_{t}^{m}(j+1) \leqslant \Delta v_{t}^{m}(j)$

(2) $\Delta v_{t+1}^{m}(j) \leqslant \Delta v_{t}^{m}(j)$.

The booking limit number $j_{1}^{m *}$ for the $m$ th class patient at the decision period $t=1$ could be given since $\Delta v_{t}^{m}(j+1) \leqslant \Delta v_{t}^{m}(j)(j \in(0, c-1))$. We define $j_{1}^{m *}$ as follows.

$$
j_{1}^{m *}= \begin{cases}j+1, & \text { if } \Delta v_{0}^{m}(j)>0 \\ \min \left(j \mid \Delta v_{0}^{m}(j) \leqslant 0\right), & \text { if } \Delta v_{0}^{m}(j) \leqslant 0 .\end{cases}
$$

$j_{1}^{m *}$ can be calculated from marginal analysis at the decision period $t=1$ in three steps.

First step: The expected marginal revenue of one more $m$ th class patient is

$$
\Delta E\left(R^{m}(j)\right)=\left(r_{m}+\pi_{m}-\gamma_{0}\right) P\left(Y_{c}+j \geqslant c\right)+\left(r_{m}+\pi_{m}+\pi_{0}\right) P\left(Y_{c}+j<c\right) .
$$

Second step: The expected marginal direct waiting cost of one more patient is obtained 
by the marginal analysis below.

- If $Y_{c} \leqslant c-j$, then there exists a critical slot $i(i \in(j, c))$ for calculating the cost of one more $m$ th class patient, which satisfies $X_{i}=0$ and $Y_{i-1}=i-j$. Before the $i$ th slot, the increased cost is $\sum_{x=1}^{i-j} e^{a(x-1)+b}$. After the $i$ th slot, the cost is $e^{a Y_{i-1}+b} P\left(X_{i+1}=\right.$ 1) $+e^{a\left(Y_{i-1}+1\right)+b} P\left(X_{i+1}=1\right) P\left(X_{i+2}=1\right)+\cdots+e^{a\left(Y_{i-1}+c-i-1\right)+b} P\left(X_{i+1}=1\right) P\left(X_{i+2}=\right.$ $1) \cdots P\left(X_{c}=1\right)$. So the expected marginal direct waiting cost is $\left\{\sum_{x=1}^{i-j} e^{a(x-1)+b}+\right.$ $\left.p_{i+1} e^{a(i-j)+b}+p_{i+1} p_{i+2} e^{a(i-j+1)+b}+p_{i+1} p_{i+2} p_{i+3} e^{a(i-j+2)+b}+\cdots+p_{i+1} p_{i+2} \cdots p_{c} e^{a(c-j-1)+b}\right\}$ $P\left(X_{i}=0\right) P\left(Y_{i-1}=i-j\right)$.

- If $Y_{c}>c-j$, the expected marginal direct waiting cost is $\sum_{x=1}^{Y_{c}} e^{a(x-1)+b} P\left(Y_{c}>c-j\right)$. So the expected marginal direct waiting cost of one more $m$ th class patient is

$$
\begin{aligned}
\Delta E(W(j))= & e^{b}\left\{\sum_{i=j}^{c}\left[\sum_{x=1}^{i-j} e^{a(x-1)}+\sum_{g=i+1}^{c} \prod_{h=i+1}^{g} p_{h} e^{a(g-j-1)}\right] P\left(X_{i}=0\right) P\left(Y_{i-1}=i-j\right)\right. \\
& \left.+\sum_{u=c-j+1}^{c} \sum_{x=1}^{u} e^{a(x-1)} P\left(Y_{c}=u\right)\right\} .
\end{aligned}
$$

$\Delta E(W(j))$ is related to the distribution of emergency patients and the reservation state $j$, independent of patient class. The marginal direct waiting cost of Example 2 is divided into two parts: $e^{b}+e^{a+b}+e^{2 a+b}$ and $e^{3 a+b}+e^{4 a+b}$. In this example, $i=7\left(X_{7}=0\right.$ and $Y_{i-1}=Y_{6}=3$, $i-j=7-4=3)$.

Third step: $\Delta E\left(R^{m}(j)\right)$ is decreasing in $j . \Delta E(W(j))$ is increasing in $j$. Accepting an $m$ th class patient's request if and only if $\Delta v_{t}^{m}(j)=\Delta E\left(R^{m}(j)\right)-\Delta E(W(j))>0$, namely,

$$
P\left(Y_{c} \leqslant c-j-1\right)>\frac{\gamma_{0}+\Delta E(W(j))-r_{m}-\pi_{m}}{\pi_{0}+\gamma_{0}}=\eta^{m}(j) .
$$

Obviously, $\eta^{m}(j)$ is increasing in $j$ and decreasing in $r_{m}+\pi_{m}$.

By solving a newsvendor-like problem in three steps, we obtain that if $j<j_{1}^{m *}, \Delta v_{0}^{m}(j)>0$; if $j \geqslant j_{1}^{m *}, \Delta v_{0}^{m}(j) \leqslant 0$. That is, the scheduler accepts a booking request if the reservation state $j$ is less than the booking limit number $j_{1}^{m *}$ of this patient. Next, we show that this booking limit number does not merely hold at decision period $t=1$ but also holds at arbitrary decision period.

Theorem 2. The booking limit numbers $j_{1}^{m *}$ obtained at the decision period $t=1$ have the following properties at arbitrary decision period $t \in(\tau, \tau-1, \ldots, 1)$ :

(1) If $j<j_{1}^{m *}$, then $\Delta v_{t}^{m}(j)>0$;

(2) If $j \geqslant j_{1}^{m *}$, then $\Delta v_{t}^{m}(j) \leqslant 0$.

Corollary 1. The incremental benefit $\Delta v_{t-1}^{m}(j)>0$ holds at an arbitrary decision period $t$ and arbitrary reservation state $j(j \in(0, c-1))$ if the booking limit number $j_{1}^{m *}=c$.

Theorem 2 shows that $j_{1}^{m *}$ could be used as the booking limit number for the $m$ th class patient at each decision period to ensure that the incremental benefit is positive. Corollary 1 shows that the request from $m$ th class $\left(j_{1}^{m *}=c\right)$ could be accepted at any decision period if there is an unscheduled slot.

The booking limit number $j_{1}^{m *}$ is related to $r_{m}+\pi_{m}, \gamma_{0}, \pi_{0}, a, b$ and the random arrival of emergency patients but independent of the call rate $\lambda_{t}$ as well as decision period $t$ from the above theorems. Theorem 2 implies the booking limit structure. Deciding to accept or delay each request simply involves comparing current reservation state to the booking limit number of that patient, and this comparison does not depend on how many requests there are in total. 
Table 1: The total revenue $r_{m}$ and penalty $\pi_{m}$ values for each patient class $m$

\begin{tabular}{|c|c|c|c|c|c|c|}
\hline \multirow{2}{*}{ Positions } & \multicolumn{2}{|l|}{$\mathrm{HP}$} & \multicolumn{2}{|l|}{ OUT } & \multicolumn{2}{|l|}{ IN } \\
\hline & $r_{m}$ & $\pi_{m}$ & $r_{m}$ & $\pi_{m}$ & $r_{m}$ & $\pi_{m}$ \\
\hline 1 & 35.21 & 1.41 & 36.62 & 4.23 & 38.03 & 14.08 \\
\hline 2 & 45.07 & 1.41 & 46.48 & 4.23 & 47.88 & 14.08 \\
\hline 3 & 54.93 & 2.82 & 56.33 & 5.63 & 57.74 & 16.90 \\
\hline 4 & 64.78 & 2.82 & 66.19 & 5.63 & 67.60 & 16.90 \\
\hline 5 & 74.62 & 2.82 & 76.05 & 5.63 & 77.46 & 16.90 \\
\hline
\end{tabular}

Once the reservation state $j$ achieves the booking limit number, his request will be delayed, no matter at which decision period.

Still, we could do more to improve the total expected reward from the second part of Proposition 1 although the booking limit numbers ensure that the incremental benefit is positive.

Corollary 2. If $r_{m}+\pi_{m} \leqslant r_{m^{\prime}}+\pi_{m^{\prime}}$, then $\Delta v_{t+1}^{m}(j) \leqslant \Delta v_{t+1}^{m^{\prime}}(j) \leqslant \Delta v_{t}^{m^{\prime}}(j)$.

This result shows that scheduler might reserve the current slot for the future use of the higher $r_{m^{\prime}}+\pi_{m^{\prime}}$ patient class although the current slot is available for the lower $r_{m}+\pi_{m}$ patient class in order to get more incremental benefit.

\section{$5 \quad$ Numerical analysis}

\section{$5.1 \quad$ Input parameters}

Parameters used in this section are based on real data from SCPH. The data in this subsection will be used as a "Base case" throughout this section.

The estimation of total revenue and penalty for delaying request of each patient class are based on the conversations with medical staff, administrator and patients. The fee is regulated by the government. The fee charged by the hospital for one position plain scanning is $€ 35.21$ (all monetary unit were converted from CNY $¥$ to Euro $€$ ), no matter which patient type (OUT, IN, $\mathrm{HP})$ it is. Adding one position per each scan charges for additional $€ 9.86$. Inpatients assist the doctors in delving into the pathophysiological process and enrich the doctors' clinical experience. The revenue of an IN is greater than both an OUT and HP if they scan the same number of positions. The revenue of a HP is the lowest since HP normally wouldn't ask for additional services. The total revenue reflects the fee and the preference of the hospital.Compared to an OUT and HP, there is a higher cost of delaying a request of an IN since it may result in longer hospital stays for inpatients. Multiple scan positions (3, 4, 5 positions) usually means serious condition of that patient. So the penalty for delaying requests of these patients is larger than patients who scan one and two positions. Table 1 shows the parameters of $r_{m}$ and $\pi_{m}$.

The capacity of each scanner consists of $c=40$ slots for 6 minutes, which equals 4 hours in each service session. There are 3 parallel scanners which contains 120 slots in one service session. The scheduler assigns each plain scanning patient one slot in practice, no matter how many positions the patient would be scanned. Reasons are listed below. First, as technology developed, multilayer spiral CT scanner spends less than 1 minute giving multi-positions' highquality images, while the early CT scanner required 3-6 minutes to give one position's lowquality image. Second, patients who have handicaps or limited mobility should turn to the central-transportation department in an effort to minimize the transportation time. There are two care workers who help patient come in and go out of the room. Third, the preparation work 


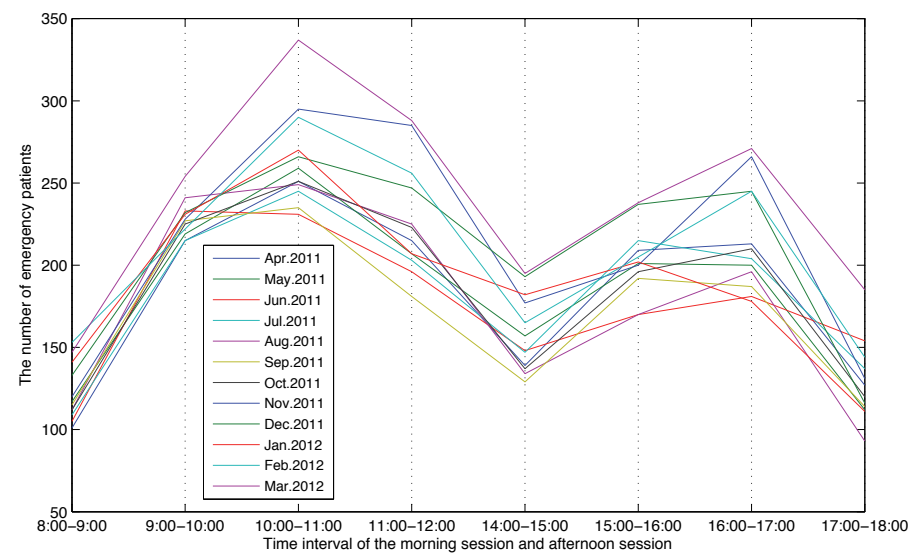

Figure 1: The number of emergency patients for each month

Table 2: The average emergency demand intensity of each scanner in March 2012

\begin{tabular}{lllll}
\hline \multicolumn{1}{c}{ Morning } & & & Afternoon & \\
\cline { 1 - 2 } \cline { 5 - 5 } time(slot) & $p_{i}$ & & time(slot) & $p_{i}$ \\
\hline 8:00-9:00 $(i \in(1,10))$ & 0.1581 & & $14: 00-15: 00(i \in(1,10))$ & 0.2097 \\
9:00-11:00 $(i \in(11,30))$ & 0.3177 & & $15: 00-17: 00(i \in(11,30))$ & 0.2737 \\
11:00-12:00 $(i \in(31,40))$ & 0.3097 & & $17: 00-18: 00(i \in(31,40))$ & 0.1989 \\
\hline
\end{tabular}

and operation procedure are uniform for plain scanning. All these measures reduce the variance of the service time of different plain scanning patients. Fourth, the service time is the time the patient occupies the $\mathrm{CT}$ room. It includes the radiation shielding door opening, patient walking into the room and lying down, radiation technologists announcing the attentions, the radiation shielding door closing, scanning time, lowering the machine and patient getting up, the radiation shielding door opening and patient coming out.

The number of emergency patients in the morning session and afternoon session from April 2011 to March 2012 is obtained by data in SCHP. They are given in Figure 1. One line represents one month. The emergency flow takes on low (8:00-9:00)-to-high (9:00-12:00) in the morning session and low at both ends (14:00-15:00, 17:00-18:00) and high in the middle (15:00-17:00) in the afternoon session in each month. In what follows, we take March 2012 for example to run the numerical analysis. The booking limit numbers can be resolved by formula (9) for different emergency demand pattern. The average emergency demand intensity of each scanner (equals to the number of emergency patients divided by the capacity) are given in Table 2 .

The overtime cost $\gamma_{0}$ and penalty for an unutilized slot $\pi_{0}$ are estimated by the cost of running the scanner (facilities, equipment and staff salaries). The fixed cost of running one scanner in a year is $€ 807,858.88$ (the depreciation of the machine $(€ 436,604.35)$, energy cost and the depreciation of the room $(€ 47,322.28)$, the maintenance cost of the machine $(€ 225,334.18)$ and the material consumption $(€ 98,588.07)$ ). So the fixed cost per slot (denote by $C_{f}$ ) is estimated by taking the ratio of the fixed cost and dividing by the number of working hours per day (8) times the number of working day in a year $(252 \times 95 \%=239,5 \%$ is the failure rate), or $C_{f}=€ 807,858.88 /(239 \times 8 \times 10)=€ 42.25$. Each patient is served by a medical team which 
Table 3: The booking limit numbers of each scanner for base case

\begin{tabular}{|c|c|c|c|c|c|c|}
\hline \multirow{2}{*}{ Positions } & \multicolumn{2}{|l|}{ HP } & \multicolumn{2}{|c|}{ OUT } & \multicolumn{2}{|l|}{ IN } \\
\hline & $\overline{\mathrm{AM}}$ & $\overline{\mathrm{PM}}$ & $\overline{A M}$ & $\overline{\mathrm{PM}}$ & $\overline{A M}$ & $\overline{\mathrm{PM}}$ \\
\hline$\overline{1}$ & 31 & 32 & 31 & 32 & 32 & $\overline{33}$ \\
\hline 2 & 31 & 33 & 31 & 33 & 32 & 34 \\
\hline 3 & 32 & 33 & 32 & 34 & 34 & 36 \\
\hline 4 & 33 & 34 & 34 & 35 & 40 & 40 \\
\hline 5 & 40 & 40 & 40 & 40 & 40 & 40 \\
\hline
\end{tabular}

contains 2 medical radiation technologists who operate scanner, two care workers who help patient come in and go out of the room, one doctor who reads photographic film and writes the report. The average overtime pay (medical team fee and hospital's management fee, denote by $\left.C_{p}\right)$ is $€ 28.17$. Thus, $\gamma_{0}$ is estimated to $C_{f}+C_{p}=€ 70.42$.

In CT department, there are 33 medical staff in charge of three CT scanners. There are additional 22 days paid vacation for the radiology department staff. So the working day in a year is $250-22=228$. The average annual salary is $€ 23,347.2$. So the average salary for one slot (denote by $C_{s}$ ) is estimated by taking the ratio of the total salary and dividing by the number of working hours per day (8) times the number of working day in one year (228), or $C_{s}=€ 23,347.2 \times 11 /(228 \times 8 \times 10)=€ 14.08$. The hospital manager have every incentive to keep these medical resources fully utilized. So there is an additional $€ 14.09$ penalty for an unutilized slot. So $\pi_{0}$ is estimated to $C_{f}+C_{s}+€ 14.09=€ 70.42$. However, " $\gamma_{0}=\pi_{0}$ " does not hold in general. We will alter these parameters in subsection 5.3.

Direct waiting cost is perhaps the most difficulty parameter to estimate. It varies from patient to patient. After discussing with patients and administrator, we use $a=0.07$ and $b=1.1$ in the exponential function as an average estimate of the direct wait cost of regular patients. If a regular patient has previously been delayed $0,10,20,30$ and 40 slots, he was told to wait for one more slot. The direct waiting cost of this additional slot is $€ 0.42, € 0.85, € 1.72$, $€ 3.46$ and $€ 6.96$, respectively. These values reflect that it is especially annoying for patient when there is excessive waiting for service.

\subsection{Computational results}

We first explicitly compute the booking limit number for the Base case by formula (9). The nested calculation was done in MATLAB as solver. Table 3 give the booking limit numbers of each scanner. AM and PM in Table 3 represents the morning session and afternoon session of March 2012, respectively.

The booking limit numbers in the afternoon session are greater than the morning session since the emergency flow in the afternoon session is smaller than the morning session. The demand intensity of emergency patients has significant impact on the booking limit number. The booking limit numbers of multiple scan positions patients are greater than that of one and two scan positions patients. It is in line with the fact that the former should be scanned as early as possible since the multiple scan positions usually means a serious condition.

The booking limit numbers in Table 3 becomes the "Base" policy in this work. The "Mean" policy reserves exactly $E\left(Y_{c}\right)$ slots for emergency demand in one service session. The "Current" policy is currently used by SCPH. One scanner is dedicated for emergency patients 
Table 4: The proportion of each patient class in March 2012

\begin{tabular}{llll}
\hline Positions & HP & OUT & IN \\
\hline 1 & 0.1817 & 0.5115 & 0.0941 \\
2 & 0.0451 & 0.0757 & 0.0379 \\
3 & 0.0124 & 0.0126 & 0.0134 \\
4 & 0.0014 & 0.0010 & 0.0091 \\
5 & 0.0000 & 0.0002 & 0.0039 \\
\hline
\end{tabular}

and the other two are used for regular patients. Namely, there are 80 slots for regular patients and 40 slots for emergency patients.

Corollary 2 implies that the total expected reward could be further improved. One simple way is to reduce the booking limit number of the lower $r_{m}+\pi_{m}$ patient class since their incremental benefit is less than the higher $r_{m^{\prime}}+\pi_{m^{\prime}}$ patient class. Therefore, some heuristic policies are designed to verify these theorems. The "Heuristic1*", "Heuristic2*" and "Heuristic $3 *$ " policy are obtained by subtracting 1, 2 and 3 of the booking limit numbers in Table 3 except multiple scan positions (3, 4, 5 positions) patient classes, respectively. "Heuristic" is abbreviated to "Heu".

We then numerically explore the performance of the above polices. Firstly, we simulate the regular requests in the booking horizon. $\tau=300$ decision periods are given. The call rate $\lambda_{t}$ is set to 0.6 in the base case (numerical comparison on different call rate will be given in $\S 5.3)$. The average requests in the booking horizon is 180 . This coincides with the fact that the demand exceeds the capacity (120 slots) in SCPH. Secondly, we should give the distribution of the booking request for each patient class $\lambda_{t}^{m}$. All the regular patients who had a plain scan in March 2012 are selected. The total number was 4,853. Table 4 describes the proportion of each patient class in March 2012. The data in Table 4 reflects the distribution of the booking request for each patient class since the "Current" policy does not discriminate among different patient classes until all the slots of the two CT scanners have been filled.

The simulation process is divided into several steps. First, a 15-by-300 matrix is generated by rand function in MATLAB to simulate the requests of 15 patient classes at 300 decision periods using the values in Table 4. Second, another matrix is generated to simulate the emergency demand of each scanner in the service session using the values in Table 2. Third, we compute the revenue and costs by the above policies using these two matrixes. Then we average the results of 5000 simulation runs. The performance measures include average total revenue $(r)$, average penalty cost for delaying requests $(\pi)$, average direct waiting cost $(W)$, average overtime cost $(\gamma)$, average penalty cost for unutilized resources $(\Pi)$, total average reward (Total), the 95\% confidence interval (CI) of the total average reward and average utilization of the machine (UTI). The total average reward is the mean value of the total reward in 5000 simulation runs. (the total reward is obtained by $r-\pi-W-\gamma-\Pi$ ). Table 5 shows the performance of base case in March 2012. Some conclusions could be given as follows.

- Our policies ("Base", "Heu1*", "Heu2*" and "Heu3*") which induced by the booking limit numbers significantly improve the total average reward, compared with the "Current" policy and the "Mean" policy.

- The confidence interval shows the stability of the total average reward.

- The "Heu" policies perform better than "Base" policy. It can be explained by Corollary 2. The "Heu" polices reduce the booking limit numbers of one and two positions patient 
Table 5: The performance measures of base case

\begin{tabular}{|c|c|c|c|c|c|c|c|c|}
\hline Policy & $r$ & $\pi$ & $W$ & $\gamma$ & $\Pi$ & Total & $\mathrm{CI}$ & UTI \\
\hline$\overline{\mathrm{AM} \text { Base }}$ & 3,929 & 384 & 320 & 803 & 24 & $2,399.0$ & {$[2,379.3 ; 2,418.6]$} & $109.2 \%$ \\
\hline AM Heu1* & 3,886 & 393 & 308 & 698 & 38 & $2,449.9$ & {$[2,431.2 ; 2,468.6]$} & $107.8 \%$ \\
\hline AM Heu2* & 3,807 & 405 & 292 & 569 & 68 & $2,473.3$ & {$[2,456.0 ; 2,490.6]$} & $105.9 \%$ \\
\hline AM Heu3* & 3,704 & 418 & 273 & 429 & 120 & $2,464.0$ & {$[2,448.6 ; 2,479.4]$} & $103.7 \%$ \\
\hline AM Mean & 3,422 & 487 & 243 & 240 & 237 & $2,214.8$ & {$[2,202.7 ; 2,226.9]$} & $100.0 \%$ \\
\hline AM Current & 3,146 & 523 & 0 & 0 & 485 & $2,137.8$ & {$[2,133.6 ; 2,142.1]$} & $94.3 \%$ \\
\hline PM Base & 4,143 & 356 & 317 & 828 & 16 & $2,625.7$ & {$[2,606.4 ; 2,645.0]$} & $109.6 \%$ \\
\hline PM Heu1* & 4,072 & 369 & 304 & 693 & 31 & $2,675.2$ & {$[2,656.9 ; 2,693.5]$} & $107.8 \%$ \\
\hline PM Heu2* & 3,993 & 381 & 291 & 560 & 58 & $2,702.3$ & {$[2,685.4 ; 2,719.2]$} & $105.9 \%$ \\
\hline PM Heu3* & 3,891 & 395 & 276 & 418 & 108 & $2,694.6$ & {$[2,679.5 ; 2,709.6]$} & $103.7 \%$ \\
\hline PM Mean & 3,540 & 471 & 243 & 184 & 277 & $2,364.5$ & {$[2,353.3 ; 2,375.6]$} & $98.9 \%$ \\
\hline PM Current & 3,146 & 523 & 0 & 0 & 795 & $1,827.8$ & {$[1,822.3 ; 1,833.4]$} & $90.6 \%$ \\
\hline
\end{tabular}

classes and keep the booking limit numbers of the multiple scan positions (3, 4, 5 positions) patient classes unchanged. Hence, the open slots for one and two positions patient classes close earlier than "Base" policy in the booking horizon. More slots are available for the higher $r_{m^{\prime}}+\pi_{m^{\prime}}$ patient classes whose incremental benefit are higher.

- "Heu $2^{*}$ " policy performs best on the total average reward. This policy is easy to put into practice. The scheduler uses the booking limit numbers in "Heu2*" policy as the threshold value for each patient class in the booking horizon and accepts request until the capacity threshold is reached or the booking horizon ends, whichever comes first.

- Moreover, it can be seen from "Heu1*" policy $\rightarrow$ "Heu3*" policy that the total average reward will decrease if the booking limit numbers of lower $r_{m}+\pi_{m}$ are reduced too many. The reason for this phenomenon is that there isn't enough requests of multiple scanning positions patients in the booking horizon. In other words, "Heu" policies are closely related to the proportion of each patient class in Table 4. Here we mention again that the booking limit numbers in Table 3 which ensure the incremental benefit is positive are independent of the call rate $\lambda_{t}\left(\lambda_{t}^{m}\right)$ as well as decision period $t$.

\subsection{Numerical comparisons}

It is very difficult to obtain accurate estimates for all the parameters. To compensate for the unreliability of some of the cost data as well as to represent a spectrum of possible actual operating situations across hospitals, some experimental investigations should be done under a fairly broad range of values around the base case. Similar experimental investigations are done in the previous works (Green et al. 2006; Gupta and Wang 2008; Kolisch and Sickinger 2008; Sickinger and Kolisch 2009). We systematically vary one of the input parameters each time, with all other parameters remaining at their base values. The range of parameters in the next experiments are also determined by discussion with medical staff, administrators and patients. In each experiment, the booking limit numbers are recalculated by formula (9). These booking limit numbers becomes the "Base*" policy in each experiment. The rest "Heu" policies are obtained in the same way as in subsection 5.2. Then, we repeat all the simulation steps in subsection 5.2 to obtain the total average reward. 
Experiment 1: $\left(\pi_{1}, \pi_{2}, \pi_{3}, \pi_{4}, \pi_{5}\right)=(0,0,0,0,0)$. There is no penalty cost for HP since some of them pays no attention to delay from one session to another. $\left(\pi_{6}, \pi_{7}, \pi_{8}, \pi_{9}, \pi_{10}\right)=$ $(1.41,1.41,2.82,2.82,2.82),\left(\pi_{11}, \pi_{12}, \pi_{13}, \pi_{14}, \pi_{15}\right)=(8.45,8.45,12.68,12.68,12.68)$. The penalty cost of outpatient and inpatient are just slightly reduced since outpatient might go to hospital repeatedly and delaying inpatients' requests will lead to longer hospital stays.

The overtime pay is influenced by the age, professional titles and educational background of the medical team. The overtime pay for a professor team is three times as an assistant team. There is no overtime pay in some hospital since it is the medical staff's responsibility to finish all the scheduled patients. So $C_{p}$ takes value of $€ 14.08$ in Experiment $2, € 0$ in Experiment 3 and $€ 42.25$ in Experiment 4, respectively.

There is no an additional penalty for an unutilized slot since the service capacity exceeds demands in some hospital. Thus, $\pi_{0}$ is estimated by $C_{f}+C_{s} . \pi_{0}$ is even only estimated by $C_{f}$ in some hospital since the salary is closely related to the number of patients he/she has served. So $\pi_{0}$ takes value of $€ 56.33$ in Experiment 5 and $€ 42.25$ in Experiment 6 , respectively. Experiment 7: $\pi_{0}$ and $\gamma_{0}$ are set back at $€ 56.33$ to combine the effects of overtime cost and penalty cost together.

The parameters $a$ and $b$ represent how sensitive patients are to the waiting time. However, the unit cost may differ from patient to patient after interview the patient and medical staff. Some patients are more sensitive to the long time waiting, such as a businessman. So we give the next two experiments to show this difference. Experiment 8: the unit direct waiting cost value is estimated by $a=0.08$ and $b=1.1$, respectively. If the patient had previously been delayed 0, 10, 20, 30 and 40 slots, he was told to wait for an additional slot. The direct waiting cost of this additional slot is $€ 0.42, € 0.94, € 2.10, € 4.66$ and $€ 10.38$, respectively. Experiment 9: $a=0.07$ and $b=1.4$. If the patient has previously been delayed $0,10,20,30$ and 40 slots, he was told to wait for an additional slot. The direct waiting cost of this additional slot is $€ 0.57$, $€ 1.15, € 2.32, € 4.66$ and $€ 9.39$, respectively.

Some patients are less sensitive to the long time waiting, such as a retiree. So we give the next two experiments to show this difference. Experiment 10: the unit direct waiting cost value is estimated by $a=0.06$ and $b=1.1$. If the patient has previously been delayed $0,10,20,30$ and 40 slots, he was told to wait for an additional slot. The direct waiting cost of this additional slot is $€ 0.42, € 0.77, € 1.4, € 2.56$ and $€ 4.66$, respectively. Experiment 11: $a=0.07$ and $b=0.9$. If a regular patient has previously been delayed $0,10,20,30$ and 40 slots, he was told to wait for an additional slot. The direct waiting cost of this additional slot is $€ 0.35, € 0.7, € 1.4, € 2.83$ and $€ 5.7$, respectively.

The number of booking requests frequently fluctuates in each service session. The scheduler estimates the interval of this variation as $(120,240)$. We expect to investigate the impact of call rate on the total expected reward in the next two experiments. Experiment 12: The call rate is set to 0.4 . So the average requests in the booking horizon is 120 , which is equal to the service capacity of one service session. Experiment 13: The call rate is set to 0.8 . So the average requests in the booking horizon is 240 , which the requests are twice as the service capacity.

The total average reward of these experiments in the morning session and afternoon session are given in Table 6 . The confidence interval (CI) of the best policy in each experiment is also listed. We conclude that all of our policies which come from booking limit structure outperform the "Current" policy and "Mean" policy on the total expected reward under different scenarios. 
Table 6: The total average reward of different experiments in March 2012

\begin{tabular}{|c|c|c|c|c|c|c|c|c|}
\hline$\overline{\text { No. }}$ & Changed parameters & Base* $^{*}$ & Heu1* & Heu2* & $\mathrm{Heu}^{*}$ & Mean & Current & $\overline{\mathrm{CI}}$ \\
\hline$\overline{\mathrm{AM} 1}$ & $\pi_{m}(m \in M)$ & 2,655 & 2,700 & 2,723 & 2,706 & 2,486 & 2,429 & {$[2,707 ; 2,740]$} \\
\hline AM2 & $\gamma_{0} \downarrow € 56.33$ & 2,538 & 2,584 & 2,619 & 2,639 & 2,263 & 2,138 & {$[2,623 ; 2,654]$} \\
\hline AM3 & $\gamma_{0} \downarrow € 42.25$ & 2,782 & 2,815 & 2,834 & 2,854 & 2,311 & 2,138 & {$[2,838 ; 2,870]$} \\
\hline AM4 & $\gamma_{0} \uparrow € 84.5$ & 2,305 & 2,360 & 2,375 & 2,349 & 2,167 & 2,138 & {$[2,357 ; 2,393]$} \\
\hline AM5 & $\pi_{0} \downarrow € 56.33$ & 2,428 & 2,470 & 2,494 & 2,481 & 2,262 & 2,235 & {$[2,477 ; 2,511]$} \\
\hline AM6 & $\pi_{0} \downarrow € 42.25$ & 2,458 & 2,503 & 2,532 & 2,527 & 2,309 & 2,332 & {$[2,515 ; 2,549]$} \\
\hline AM7 & $\gamma_{0}, \pi_{0} \downarrow € 56.33$ & 2,572 & 2,616 & 2,644 & 2,652 & 2,310 & 2,235 & {$[2,637 ; 2,667]$} \\
\hline AM8 & $a \uparrow 0.08$ & 2,382 & 2,433 & 2,458 & 2,450 & 2,203 & 2,138 & {$[2,440 ; 2,476]$} \\
\hline AM9 & $b \uparrow 1.4$ & 2,312 & 2,355 & 2,378 & 2,359 & 2,130 & 2,138 & {$[2,360 ; 2,396]$} \\
\hline AM10 & $a \downarrow 0.06$ & 2,415 & 2,465 & 2,488 & 2,477 & 2,226 & 2,138 & {$[2,471 ; 2,504]$} \\
\hline AM11 & $b \downarrow 0.9$ & 2,451 & 2,501 & 2,524 & 2,514 & 2,259 & 2,138 & {$[2,507 ; 2,541]$} \\
\hline AM12 & $\lambda_{t} \downarrow 0.4$ & 2,684 & 2,703 & 2,685 & 2,619 & 2,528 & 2,451 & {$[2,686 ; 2,720]$} \\
\hline AM13 & $\lambda_{t} \uparrow 0.8$ & 2,107 & 2,167 & 2,217 & 2,244 & 1,900 & 1,823 & {$[2,226 ; 2,261]$} \\
\hline PM1 & $\pi_{m}(m \in M)$ & 2,843 & 2,894 & 2,914 & 2,895 & 2,627 & 2,119 & {$[2,898 ; 2,930]$} \\
\hline PM2 & $\gamma_{0} \downarrow € 56.33$ & 2,751 & 2,797 & 2,830 & 2,845 & 2,401 & 1,828 & {$[2,830 ; 2,861]$} \\
\hline PM3 & $\gamma_{0} \downarrow € 42.25$ & 2,986 & 3,023 & 3,040 & 3,053 & 2,438 & 1,828 & {$[3,039 ; 3,068]$} \\
\hline PM4 & $\gamma_{0} \uparrow € 84.5$ & 2,513 & 2,565 & 2,589 & 2,562 & 2,328 & 1,828 & {$[2,571 ; 2,606]$} \\
\hline PM5 & $\pi_{0} \downarrow € 56.33$ & 2,633 & 2,685 & 2,717 & 2,718 & 2,420 & 1,987 & {$[2,703 ; 2,733]$} \\
\hline PM6 & $\pi_{0} \downarrow € 42.25$ & 2,638 & 2,689 & 2,712 & 2,708 & 2,475 & 2,146 & {$[2,696 ; 2,729]$} \\
\hline PM7 & $\gamma_{0}, \pi_{0} \downarrow € 56.33$ & 2,755 & 2,801 & 2,837 & 2,854 & 2,457 & 1,987 & {$[2,839 ; 2,870]$} \\
\hline PM8 & $a \uparrow 0.08$ & 2,611 & 2,661 & 2,689 & 2,682 & 2,354 & 1,828 & {$[2,672 ; 2,706]$} \\
\hline PM9 & $b \uparrow 1.4$ & 2,514 & 2,569 & 2,600 & 2,598 & 2,279 & 1,828 & {$[2,582 ; 2,619]$} \\
\hline PM10 & $a \downarrow 0.06$ & 2,639 & 2,688 & 2,715 & 2,706 & 2,374 & 1,828 & {$[2,698 ; 2,619]$} \\
\hline PM11 & $b \downarrow 0.9$ & 2,681 & 2,727 & 2,754 & 2,745 & 2,409 & 1,828 & {$[2,738 ; 2,770]$} \\
\hline PM12 & $\lambda_{t} \downarrow 0.4$ & 2,899 & 2,915 & 2,894 & 2,822 & 2,677 & 2,141 & {$[2,899 ; 2,931]$} \\
\hline PM13 & $\lambda_{t} \uparrow 0.8$ & 2,325 & 2,388 & 2,441 & 2,473 & 2,050 & 1,513 & {$[2,456 ; 2,490]$} \\
\hline
\end{tabular}




\section{Discussion}

The booking problem for CT department is modeled as a MDP in this work. To the best of our knowledge, a unique feature of our model is mathematically modelling the direct waiting cost of regular patients in the service session under the random arrival of emergency patients. Some structural properties are given to obtain the booking limit numbers in the booking horizon. These booking limit numbers are proved to be independent of the call rate and the decision period. Therefore, the booking limit numbers are easy to implement in the booking horizon. At any time in the booking horizon, deciding to accept or delay each request simply involves comparing current reservation state to the booking limit number of that patient. These booking limit numbers yield substantial improvement on the total expected reward over the policy currently employed.

However, more works remains to be done. One potential complication that we are currently studying is the variation of the service time among different patients. Mathematically description the direct waiting cost in advance scheduling is a challenge work. Furthermore, the penalty for delaying the booking request may be related to the time span between his preferred service session and his successfully booked service session-a characteristic we could not incorporate into our single service session framework. These indicate the need of future research in these directions.

\section{A Appendix}

\section{A.1 Proof of Lemma 1}

$$
\begin{aligned}
H\left(Y_{c}, j+1\right)-H\left(Y_{c}, j\right)= & -\pi_{0}\left(c-j-1-Y_{c}\right)^{+}-\gamma_{0}\left(Y_{c}+j+1-c\right)^{+} \\
& +\pi_{0}\left(c-j-Y_{c}\right)^{+}+\gamma_{0}\left(Y_{c}+j-c\right)^{+} \\
H\left(Y_{c}, j\right)-H\left(Y_{c}, j-1\right)= & -\pi_{0}\left(c-j-Y_{c}\right)^{+}-\gamma_{0}\left(Y_{c}+j-c\right)^{+} \\
& +\pi_{0}\left(c+1-j-Y_{c}\right)^{+}+\gamma_{0}\left(Y_{c}+j-1-c\right)^{+} .
\end{aligned}
$$

We look at four separate cases: $1 . c-j-Y_{c} \leqslant-1 ; 2 .-1<c-j-Y_{c} \leqslant 0 ; 3.0<c-j-Y_{c} \leqslant 1$; 4. $c-j-Y_{c}>1$.

1. For the case $c-j-Y_{c} \leqslant-1$, (10) becomes

$H\left(Y_{c}, j+1\right)-H\left(Y_{c}, j\right)=-\gamma_{0} ; H\left(Y_{c}, j\right)-H\left(Y_{c}, j-1\right)=-\gamma_{0}$

$H\left(Y_{c}, j+1\right)-H\left(Y_{c}, j\right)=H\left(Y_{c}, j\right)-H\left(Y_{c}, j-1\right)$;

2. For the case $-1<c-j-Y_{c} \leqslant 0$, that is $c-j-Y_{c}=0$, (10) becomes

$H\left(Y_{c}, j+1\right)-H\left(Y_{c}, j\right)=-\gamma_{0} ; H\left(Y_{c}, j\right)-H\left(Y_{c}, j-1\right)=\pi_{0}$;

$H\left(Y_{c}, j+1\right)-H\left(Y_{c}, j\right) \leqslant H\left(Y_{c}, j\right)-H\left(Y_{c}, j-1\right)$;

3. For the case $0<c-j-Y_{c} \leqslant 1$, that is $c-j-Y_{c}=1$, (10) becomes

$H\left(Y_{c}, j+1\right)-H\left(Y_{c}, j\right)=\pi_{0} ; H\left(Y_{c}, j\right)-H\left(Y_{c}, j-1\right)=\pi_{0} ;$

$H\left(Y_{c}, j+1\right)-H\left(Y_{c}, j\right)=H\left(Y_{c}, j\right)-H\left(Y_{c}, j-1\right)$;

4. For the case $c-j-Y_{c}>1$, (10) becomes

$H\left(Y_{c}, j+1\right)-H\left(Y_{c}, j\right)=\pi_{0} ; H\left(Y_{c}, j\right)-H\left(Y_{c}, j-1\right)=\pi_{0} ;$

$H\left(Y_{c}, j+1\right)-H\left(Y_{c}, j\right)=H\left(Y_{c}, j\right)-H\left(Y_{c}, j-1\right)$.

Thus $H\left(Y_{c}, j\right)$ is concave in $j$. This completes the proof. 


\section{A.2 Proof of Lemma 2}

$$
\begin{aligned}
& W_{i}\left(Y_{i}, X_{i}, j+1\right)-W_{i}\left(Y_{i}, X_{i}, j\right)= M_{i}(j+1) \cdot X_{i} \cdot\left(j+1-i+1+Y_{i}-X_{i}\right) \cdot e^{a Y_{i-1}+b} \\
&-M_{i}(j) \cdot X_{i} \cdot\left(j-i+1+Y_{i}-X_{i}\right) \cdot e^{a Y_{i-1}+b} \\
& \begin{aligned}
W_{i}\left(Y_{i}, X_{i}, j\right)-W_{i}\left(Y_{i}, X_{i}, j-1\right)= & M_{i}(j) \cdot X_{i} \cdot\left(j-i+1+Y_{i}-X_{i}\right) \cdot e^{a Y_{i-1}+b} \\
& -M_{i}(j-1) \cdot X_{i} \cdot\left(j-1-i+1+Y_{i}-X_{i}\right) \cdot e^{a Y_{i-1}+b} .
\end{aligned}
\end{aligned}
$$

We look at four separate cases: $1 . Y_{i} \leqslant i-j-1 ; 2 . i-j-1<Y_{i} \leqslant i-j ; 3 . i-j<Y_{i} \leqslant i-j+1$; 4. $Y_{i}>i-j+1$.

1. For the case $Y_{i} \leqslant i-j-1,(11)$ becomes

$$
\begin{aligned}
& W_{i}\left(Y_{i}, X_{i}, j+1\right)-W_{i}\left(Y_{i}, X_{i}, j\right)=0 ; W_{i}\left(Y_{i}, X_{i}, j\right)-W_{i}\left(Y_{i}, X_{i}, j-1\right)=0 \\
& W_{i}\left(Y_{i}, X_{i}, j+1\right)-W_{i}\left(Y_{i}, X_{i}, j\right)=W_{i}\left(Y_{i}, X_{i}, j\right)-W_{i}\left(Y_{i}, X_{i}, j-1\right) ;
\end{aligned}
$$

2. For the case $i-j-1<Y_{i} \leqslant i-j$, that is, $Y_{i}=i-j$, (11) becomes

$$
W_{i}\left(Y_{i}, X_{i}, j+1\right)-W_{i}\left(Y_{i}, X_{i}, j\right)=X_{i} e^{a Y_{i-1}+b}\left(2-X_{i}\right) ; W_{i}\left(Y_{i}, X_{i}, j\right)-W_{i}\left(Y_{i}, X_{i}, j-1\right)=0 ;
$$$$
W_{i}\left(Y_{i}, X_{i}, j+1\right)-W_{i}\left(Y_{i}, X_{i}, j\right) \geqslant W_{i}\left(Y_{i}, X_{i}, j\right)-W_{i}\left(Y_{i}, X_{i}, j-1\right) \text {; }
$$

3. For the case $i-j<Y_{i} \leqslant i-j+1$, that is, $Y_{i}=i-j+1$, (11) becomes

$$
\begin{aligned}
& W_{i}\left(Y_{i}, X_{i}, j+1\right)-W_{i}\left(Y_{i}, X_{i}, j\right)=X_{i} e^{a Y_{i-1}+b} ; W_{i}\left(Y_{i}, X_{i}, j\right)-W_{i}\left(Y_{i}, X_{i}, j-1\right)=X_{i} e^{a Y_{i-1}+b}(2- \\
& \left.X_{i}\right) ; \\
& W_{i}\left(Y_{i}, X_{i}, j+1\right)-W_{i}\left(Y_{i}, X_{i}, j\right)=W_{i}\left(Y_{i}, X_{i}, j\right)-W_{i}\left(Y_{i}, X_{i}, j-1\right) ;
\end{aligned}
$$

4. For the case $Y_{i}>i-j+1$, (11) becomes

$$
\begin{aligned}
& W_{i}\left(Y_{i}, X_{i}, j+1\right)-W_{i}\left(Y_{i}, X_{i}, j\right)=X_{i} e^{a Y_{i-1}+b} ; W_{i}\left(Y_{i}, X_{i}, j\right)-W_{i}\left(Y_{i}, X_{i}, j-1\right)=X_{i} e^{a Y_{i-1}+b} ; \\
& W_{i}\left(Y_{i}, X_{i}, j+1\right)-W_{i}\left(Y_{i}, X_{i}, j\right)=W_{i}\left(Y_{i}, X_{i}, j\right)-W_{i}\left(Y_{i}, X_{i}, j-1\right) .
\end{aligned}
$$

Thus $-W_{i}\left(Y_{i}, X_{i}, j\right)$ is concave in $j$. A weight average (nonnegative weights) of concave function is also concave function since $-E\left(W_{i}(j)\right)=E_{Y_{i}, X_{i}}\left(-W_{i}\left(Y_{i}, X_{i}, j\right)\right)$. Hence it follows that $-E\left(W_{i}(j)\right)$ is concave in $j$ as well. $-E(W(j))$ is also concave in $j$. We have proved that $H\left(Y_{c}, j\right)$ is concave in $j . E_{Y_{c}}\left(H\left(Y_{c}, j\right)\right)$ is concave as well since it is a weight average (nonnegative weights) of concave function. Then $v_{0}(j)$ is concave in $j$. This completes the proof.

\section{A.3 Proof of Lemma 3}

The proof of the convexity of $v_{t}(j)$ in $j$ for $\forall t$ is based on the work by Lautenbacher and Stidham(1999).

$$
f(j)=\max \left\{v(j+1)+r_{m}, v(j)-\pi_{m}\right\}=\max _{0 \leqslant u \leqslant 1, u \in Z}\left\{v(j+u)+u\left(r_{m}+\pi_{m}\right)\right\}-\pi_{m} .
$$

By changing variables to $y=j+u$ we can write $f(j)=\widehat{f}(j)-j\left(r_{m}+\pi_{m}\right)-\pi_{m}$, where

$$
\widehat{f}(j)=\max _{j \leqslant y \leqslant j+1, y \in Z}\left\{v(y)+y\left(r_{m}+\pi_{m}\right)\right\} .
$$

Let $y^{*}=\operatorname{argmax}_{y \geqslant 0}\left\{v(y)+y\left(r_{m}+\pi_{m}\right)\right\} . v(y)+y\left(r_{m}+\pi_{m}\right)$ is concave since $v(y)$ is concave. Moreover, $v(y)+y\left(r_{m}+\pi_{m}\right)$ is nondecreasing for $y \leqslant y^{*}$ and nonincreasing for $y>y^{*}$.

If $j \leqslant y^{*}-2, \widehat{f}(j+1)-\widehat{f}(j)=v(j+2)-v(j+1)+r_{m}+\pi_{m} \leqslant v(j+1)-v(j)+r_{m}+\pi_{m}=$ $\widehat{f}(j)-\widehat{f}(j-1)$.

If $j=y^{*}-1, \widehat{f}(j+1)-\widehat{f}(j)=v(j+1)-v(j+1)=0, \widehat{f}(j)-\widehat{f}(j-1) \geqslant 0$.

If $j=y^{*}, \widehat{f}(j+1)-\widehat{f}(j) \leqslant 0, \widehat{f}(j)-\widehat{f}(j-1)=0$.

If $j \geqslant y^{*}+1, \widehat{f}(j+1)-\widehat{f}(j)=v(j+1)-v(j)+r_{m}+\pi_{m} \leqslant v(j)-v(j-1)+r_{m}+\pi_{m}=$ $\widehat{f}(j)-\widehat{f}(j-1)$. 
Thus, $\widehat{f}(j)$ is concave in $j \geqslant 0$ and $f(j)$ is concave in $j \geqslant 0$ as well. This completes the proof. A.4 Proof of Theorem 1

From Lemma $2, v_{0}(j)$ is concave in $j$. That is, $v_{t-1}(j)$ is concave in $j$ at $t=1$. Assume that the result holds for some $t$. That is, $v_{t-1}(j)$ is concave in $j$ at $t$. The proof can be completed by showing the result also holds for $t+1$. Note that $v_{t}(j)=\sum_{m=1}^{M} \lambda_{t}^{m} \max \left\{v_{t-1}(j+1)+\right.$ $\left.r_{m}, v_{t-1}(j)-\pi_{m}\right\}+\left(1-\sum_{m=1}^{M} \lambda_{t}^{m}\right) v_{t-1}(j)$. From lemma 3 and the fact that the weighted average (nonnegative weights) of concave functions is also concave, $v_{t-1}(j)$ is concave in $j$ at $t+1$. That is, $v_{t}(j)$ is concave in $j$ at $t$. So $\Delta v_{t}^{m}(j+1) \leqslant \Delta v_{t}^{m}(j)$ holds for $\forall j, t$.

A.5 Proof of Proposition 1

(1) The first part is obtained immediately from Theorem 1.

(2) The second part

$$
\begin{aligned}
\Delta v_{t}^{m}(j) & =v_{t}(j+1)-v_{t}(j)+r_{m}+\pi_{m} \\
& =\sum_{m=1}^{M} \lambda_{t}^{m} \max \left\{\Delta v_{t-1}^{m}(j+1), 0\right\}-\sum_{m=1}^{M} \lambda_{t}^{m} \max \left\{\Delta v_{t-1}^{m}(j), 0\right\}+\Delta v_{t-1}^{m}(j) \\
& \leqslant \Delta v_{t-1}^{m}(j) .
\end{aligned}
$$

\section{A.6 Proof of Theorem 2}

We have proved that if $j<j_{1}^{m *}, \Delta v_{0}^{m}(j)>0$; if $j \geqslant j_{1}^{m *}, \Delta v_{0}^{m}(j) \leqslant 0$. Assume that the result holds for some $t$. That is, if $j<j_{1}^{m *}, \Delta v_{t-1}^{m}(j)>0$; if $j \geqslant j_{1}^{m *}, \Delta v_{t-1}^{m}(j) \leqslant 0$.

The proof is completed by showing that the result also holds for $t+1$.

$$
\Delta v_{t}^{m}(j)=\sum_{m=1}^{M} \lambda_{t}^{m} \max \left\{\Delta v_{t-1}^{m}(j+1), 0\right\}-\sum_{m=1}^{M} \lambda_{t}^{m} \max \left\{\Delta v_{t-1}^{m}(j), 0\right\}+\Delta v_{t-1}^{m}(j) .
$$

1. If $j<j_{1}^{m *}-1$, then $j+1<j_{1}^{m *}, \Delta v_{t-1}^{m}(j+1)>0, \Delta v_{t-1}^{m}(j)>0$, (12) becomes

$$
\Delta v_{t}^{m}(j)=\sum_{m=1}^{M} \lambda_{t}^{m} \Delta v_{t-1}^{m}(j+1)+\left(1-\sum_{m=1}^{M} \lambda_{t}^{m}\right) \Delta v_{t-1}^{m}(j)>0 .
$$

2. If $j=j_{1}^{m *}-1$, then $j+1=j_{1}^{m *}, \Delta v_{t-1}^{m}(j+1) \leqslant 0, \Delta v_{t-1}^{m}(j)>0$, (12) becomes

$$
\Delta v_{t}^{m}(j)=\left(1-\sum_{m=1}^{M} \lambda_{t}^{m}\right) \Delta v_{t-1}^{m}(j)>0 \text {. }
$$

3. If $j \geqslant j_{1}^{m *}$, then $j+1>j_{1}^{m *}, \Delta v_{t-1}^{m}(j+1) \leqslant 0, \Delta v_{t-1}^{m}(j) \leqslant 0$, (12) becomes

$$
\Delta v_{t}^{m}(j)=\Delta v_{t-1}^{m}(j) \leqslant 0 .
$$

This completes the proof.

\section{References}

[1] Carmon Z, George SJ, Carmon T (1995) A Psychological Perspective on Service Segmentation Models: The Significance of Accountingfor Consumers' Perceptions of Waiting and Service. Management Science 41:1806-1815 
[2] Dobson G, Hasija S, Pinker E (2011) Reserving Capacity for Urgent Patients in Primary Care. Production and operations management 20:456-473

[3] Green L, Savin S, Wang B (2006) Managing patient demand in a diagnostic medical facility. Operations Research 54:11-25

[4] Gupta D, Denton B (2008) Appointment scheduling in health care: Challenges and opportunities. IIE Transactions 40:800-819

[5] Gupta D, Wang L (2008) Revenue management for a primary-care clinic in the presence of patient choice. Operations Research 56:576-592

[6] Ittig PT (1994) Planning service capacity when demand is sensitive to delay. Decision Sciences 25(4):541-553

[7] Kolisch R, Sickinger S (2008) Providing radiology health care services to to stochastic demand of different patient classes. OR Spectrum 30(2):375-395

[8] Lautenbacher CJ, Stidham SJ (1999) The underlying Markov decision process in the singleleg airline yield mamagement problem. Transportation Science 34:136-146

[9] Luo J, Kulkarni V, Ziya S (2012) Appointment scheduling under patient no-shows and service interruptions. Manufacturing and service operations management 14:670-684

[10] Magerlein JM, Martin JB (1978) Surgical demand scheduling: A review. Health Services Research 13:418-433

[11] Osuna E (1985) The Psychological Cost of Waiting. Journal of Mathematical Psychology $29: 82-105$

[12] Patrick J, Puterman ML, Queyranne M (2008) Dynamic multipriority patient scheduling for a diagnostic resource. Operations Research 56:1507-1525

[13] Sickinger S, Kolisch R (2009) The performance of a generalized Bailey-Welch rule for outpatient appointment scheduling under inpatient and emergency demand. Health Care Management Science 12(4):408-419 